\title{
THE SINGULAR PERTURBATION LIMIT OF AN ELASTIC STRUCTURE IN A RAPIDLY FLOWING NEARLY INVISCID FLUID
}

\author{
BY \\ ZVI ARTSTEIN (Department of Mathematics, The Weizmann Institute of Science, Rehovot \\ 76100, Israel) \\ AND \\ MARSHALL SLEMROD (Center for Mathematical Sciences, University of Wisconsin-Madison, \\ Madison, WI)
}

\begin{abstract}
The effective forces that govern the vertical oscillations of an elastic structure in a horizontally flowing fluid are displayed for the limit case where fluid velocity and viscosity tend to singular limits. The derived singular perturbation model is based on available representations that model the coupled dynamics as coupled oscillators. The fast dynamics of the system does not, in general, converge on the fast time scale to a stationary point; thus, the classical singular perturbation methods are not applicable. Rather, a method based on Young measures representation of the fast oscillations, and on averaging, is employed.
\end{abstract}

1. Introduction. It has long been observed that oscillating vortex forces induced by vortex shedding by an elastically mounted cylinder and horizontally flowing fluid, affect and cause vertical oscillations of both the cylinder and the fluid. The phenomenon has been modeled as coupled oscillators, with parameters interpreted in terms of physical characteristics of the fluid structure. In this paper we consider the limit behavior of the oscillations when the velocity of the fluid is high and the viscosity is small. In particular, we compute the effective forces on the elastically mounted cylinder, and the limit of the fluid oscillations, as the velocity and viscosity tend to the singular limits. We find that the vortex forces generate an effective new mass of the cylinder that is greater than the

Received February 18, 2000.

2000 Mathematics Subject Classification. Primary 34E15, 34C29, 34C41, 34C60, 74F 10.

The first author is the incumbent of the Hettie H. Heineman Professorial Chair. Research supported by a grant from the United-States-Israel Binational Science Foundation (BSF), and by the MINERVA Foundation.

The second author's research was supported by a grant from the United-States-Israel Binational Science Foundation (BSF), by the U. S. National Science Foundation Grant No. DMS-9531845, and by the Office of Naval Research No. N00014-93-0015.

E-mail address: zvika@wisdom.weizmann.ac.il

E-mail address: slemrod@cms.wisc.edu

(C)2001 Brown University 
original mass, thus shifting the cylinder closer to the instability region. In one case, the effective damping of the cylinder increases as well. We find that, depending on a chosen function parameter, the fluid either oscillates at the same pace as the cylinder, or exhibits nontrivial fast oscillations which are centered around a point that oscillates at the same pace as the cylinder.

A prime tool of our analysis is to model fast oscillations of the fluid oscillator as Young measures. Indeed, the singular perturbation system depicting the coupled fluidstructure oscillators has a fast variable that does not converge on the fast time scale to an equilibrium. Thus, the standard singular perturbation analysis, which follows the Levinson-Tichonov approach, is not applicable. We employ a recent method which represents the limit of the fast dynamics as an invariant measure on the fast time scale, resulting in a Young measure. The coupling with the slow dynamics is then done via averaging.

The paper is organized as follows. The model is developed in the next section, following the steps in Iwan and Belvins [5]. The singular perturbation limit is derived in Section 3. The analysis of the singular perturbation system is carried out in Section 4. The interpretation of the outcome in terms of the effective dynamics of the fluid-structure is displayed in the closing section.

2. The Equations of Motion. The model analyzed in this paper is a variant of models available in the literature. For completeness, and for the interpretation of the analytical results which follow, we display here the main steps of the derivation of the model. We follow the description of fluid-structure motion given by Iwan and Belvins [5], which relates to an earlier derivation of Chen [3]. Many of the basic ideas go back to von Kármán [6] in his study of vortex shedding. An excellent discussion of von Kármán's work can be found in Kochin, $\mathrm{Kibel}^{\prime}$, and Roze [7]. A model similar to what we follow has been devised by Dowell and Ilgamov [4, p. 186] from phenomenological arguments.

The model consists of an elastically mounted rigid cylinder in a horizontally flowing fluid as shown in Fig. 1.

The basic assumptions are similar to those in Iwan and Belvins [5, p. 582] and read as follows:

(i) The flow is two-dimensional, incompressible with constant density $\rho$.

(ii) The flow reaches the area of the rigid cylinder with a constant horizontal velocity $U$.

(iii) Inviscid flow provides a good approximation for the flow outside the near wake.

(iv) The flow over the cylinder generates a well-formed vortex street with a shedding frequency $\omega_{s}$.

(v) Vorticity is generated in the near wake of the cylinder while vortices grow uniformly to a maximum strength and move downstream.

(vi) The force exerted transversally on the cylinder by the fluid flow depends on the average vertical acceleration and the average vertical velocity of the fluid relative to the cylinder, near the cylinder; this force is denoted by $F_{y}$.

As explained in the introduction, we examine later the singular perturbation limit equations for high velocity $U$ and small viscosity. In this section we derive the equations 


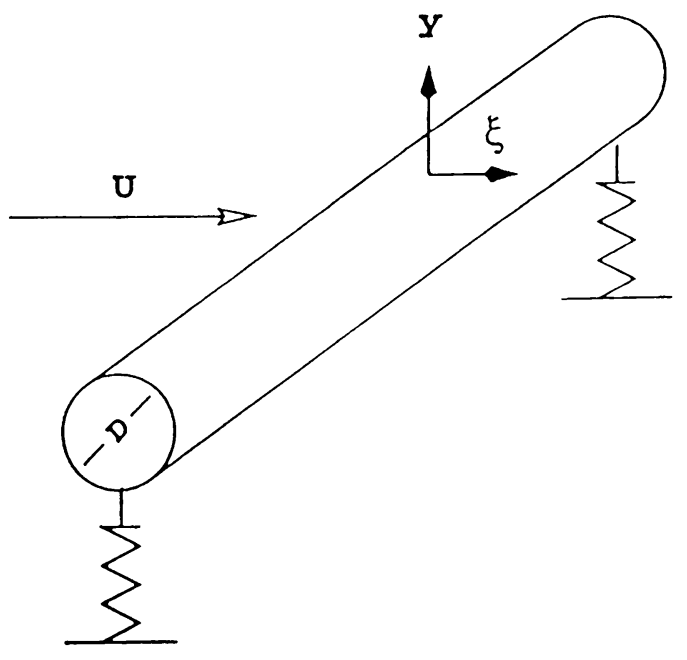

FIG. 1

of motion for a point mass representing the cylinder and for a "hidden" variable whose dynamics represent the averaged vertical velocity and acceleration of the fluid mentioned in (vi). The arguments follow Iwan and Belvins [5] with some minor modifications; see more details in [5] and references therein.

The horizontal space variable is denoted by $\xi$ and the vertical space variable by $y$. Consider the control area depicted in Fig. 2.

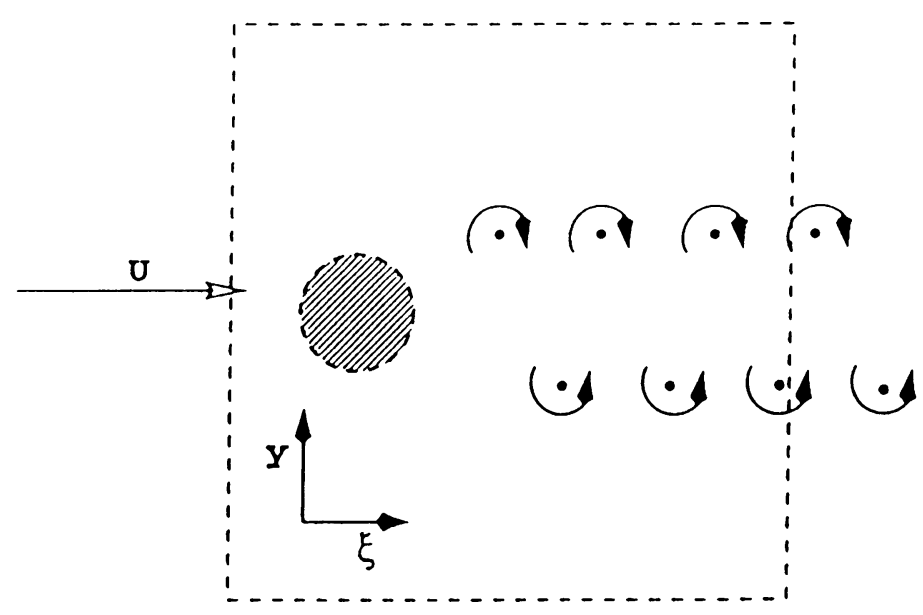

FIG. 2

Let the fluid velocity be $v(\xi, y, t)=\left(v_{\xi}(\xi, y, t), v_{y}(\xi, y, t)\right)$; it is two-dimensional as postulated in (i). We wish to compute the rate of change of the $y$-component of the fluid 
momentum, namely to compute $\frac{d}{d t} J_{y}(t)$ where

$$
J_{y}(t)=\iint \rho v_{y}(\xi, y, t) d \xi d y
$$

with the integration performed over the control area with the cylinder deleted.

Note the obvious decomposition

$$
\frac{d}{d t} J_{y}(t)=\frac{d}{d t} J_{y}^{I}(t)+\frac{d}{d t} J_{y}^{E}(t)
$$

where the superscripts $I$ and $E$ stand for, respectively, Inside and Enter/Exit. Namely, $\frac{d}{d t} J_{y}^{I}$ is the rate of change of the momentum inside the control area, while $\frac{d}{d t} J_{y}^{E}$ is the rate of change due to flow entering (or exiting) the control area. In what follows, explicit expressions are derived for the two components of the rate of change in (2.2).

First, consider the term $\frac{d}{d t} J_{y}^{E}(t)$. We follow Iwan and Belvins [5, (5)] and Chen [3] who, based on arguments similar to von Kármán's derivation of the vortex motion in an ideal fluid (see Kochin et al. [7]), concluded that

$$
\frac{d}{d t} J_{y}^{E}(t)=-\rho u_{T} \Gamma+\text { correction term }
$$

where $u_{T}$ is the transversal velocity of the vortex street and $\Gamma$ is the circulation of each vortex. Next, we take Iwan and Belvins [5, (6) and (8)] who showed, employing the experimental observation that the fluid oscillates at the rate of the vortex shedding, that $\Gamma$ can be expressed as

$$
\Gamma=K \omega_{s} D z(t)
$$

where $K$ is a constant, $\omega_{s}$ is the frequency of the vortex shedding, $D$ is the characteristic length of the cylinder cross section, and $z(t)$ is a "hidden" variable that models the displacement from equilibrium of a lumped position of the fluid particles. Inserting (2.4) into (2.3) yields

$$
\frac{d}{d t} J_{y}^{E}(t)=-K \rho u_{T} \omega_{s} D z(t)+\text { correction term. }
$$

We determine now the form of the correction term in (2.5). Invariance with respect to a sign change of the $z$-variable suggests that the correction term in (2.5), when expressed in terms of the $z$-variable, should be an odd function of $\dot{z}$ (here and throughout, a dot above a variable denotes time derivative). Accordingly, Iwan and Belvins [5, (8)] chose as a correction term a cubic function

$$
A_{1} \rho U D \dot{z}-A_{2} \rho D \frac{\dot{z}^{3}}{U}
$$

where $A_{1}$ and $A_{2}$ are positive constants. In a similar situation, Dowell and Ilgamov [4, p. 191] chose as a correction term a 7th order odd polynomial which. in our notation, reads as

$$
\rho D U^{2}\left(A_{1} \frac{\dot{z}}{U}-A_{2} \frac{\dot{z}^{3}}{U^{3}}+A_{5} \frac{\dot{z}^{5}}{U^{5}}-A_{7} \frac{\dot{z}^{7}}{U^{7}}\right) .
$$

In both cases (2.6) and (2.7), the correction term is compatible with a van der Pol type of oscillatory behavior of the fluid variable. In the present paper we choose a more general 
correction term which allows both the van der Pol oscillations and stable dynamics. Specifically, consider the term

$$
K_{1} U^{2} F\left(\frac{\dot{z}}{U}\right)
$$

with $K_{1}$ a positive constant, and $F(\eta)$ a function satisfying the following conditions.

Assumption 2.1. $F: R \rightarrow R$ satisfies

1. $F$ is an odd function, namely $F(-\eta)=-F(\eta)$;

2. $F(\eta) \rightarrow-\infty$ as $\eta \rightarrow+\infty$;

3. $F(\eta)$ is differentiable, and $\left|F^{\prime}(\eta)\right|$ is bounded away from 0 for $|\eta|$ large enough.

REMARK 2.2. Note that we do not place an assumption on the derivative of $F$ at $\eta=$ 0. Both correction terms (2.6) suggested by Iwan and Belvins [5], and (2.7) used by Dowell and Ilgamov [4], satisfy $F^{\prime}(0)>0$. In the conclusions in Sec. 5 we examine the consequences of placing an assumption on the sign of $F$ at the origin.

Employing (2.8) as the correction term in (2.5) yields

$$
\frac{d}{d t} J_{y}^{E}(t)=-K \rho u_{T} \omega_{s} D z(t)+K_{1} U^{2} F\left(\frac{\dot{z}}{U}\right) .
$$

Next, consider the term $\frac{d}{d t} J_{y}^{I}(t)$. By Newton's law, the rate of change of momentum inside the control area is equal to the sum of forces on the fluid in the area. These forces are the pressure gradient of the fluid and the force exerted on the fluid by the cylinder. Hence we write

$$
\frac{d}{d t} J_{y}^{I}(t)=P_{y}-F_{y}
$$

where $P_{y}$ is the $y$-component of the pressure force, and $-F_{y}$ the force exerted by the cylinder on the fluid, this since in (vi) we denoted by $F_{y}$ the force exerted by the fluid on the cylinder.

We claim that $P_{y}$ is approximately zero. This can be explained by a symmetry argument, or as Iwan and Belvins $[5,(4)]$ deduce it, by considering a control area with very long vertical length, then using Bernoulli's equation, with the observation that the far field flow induced by the vortex shedding diminishes at the rate of the distance from the vortex street.

For $F_{y}$ in $(2.10)$ we follow Iwan and Belvins $[5,(9)]$ and use the form

$$
F_{y}=a_{1} \rho D^{2}(\ddot{z}-\ddot{y})+a_{2} \rho D U(\dot{z}-\dot{y})
$$

with $a_{1}$ and $a_{2}$ positive constants, and $y(t)$ measuring the displacement of the cylinder from equilibrium. The first term on the right-hand side of (2.11) reflects the force due to the difference of accelerations of the fluid and the cylinder, while the second term is due to the viscosity of the fluid (thus, $a_{2}=0$ for an inviscid fluid).

By now the expressions for the right-hand side terms of (2.12) are completed. Substituting $P_{y}=0$ and the expression (2.11) into (2.10), and inserting (2.10) and (2.9) into 
(2.2), yields the equation

$$
\frac{d}{d t} J_{y}(t)=-a_{1} \rho D^{2}(\ddot{z}-\ddot{y})-a_{2} \rho D U(\dot{z}-\dot{y})-K \rho u_{T} \omega_{s} D z(t)+K_{1} U^{2} F\left(\frac{\dot{z}}{U}\right) .
$$

At this point we recall that $J_{y}(t)$ is a component of the fluid momentum, and thus it is a rate proportional to the velocity of the averaged fluid variable. Thus we write

$$
J_{y}(t)=a_{0} \rho D^{2} \dot{z}(t)
$$

compare with Iwan and Belvins [5, (3)]. In fact, (2.13) can be interpreted as the definition of the velocity of the "hidden" fluid variable, which in turn appears in the representation of $\Gamma$ in (2.4)

Inserting the time derivative of the expression in (2.13) in the left-hand side of $(2.12)$ yields a second-order equation for the lumped fluid particle as follows:

$$
\ddot{z}+\frac{a_{2} U}{\left(a_{0}+a_{1}\right) D} \dot{z}-\frac{K_{1} U^{2}}{\left(a_{0}+a_{1}\right) \rho D^{2}} F\left(\frac{\dot{z}}{U}\right)+\frac{K u_{T} \omega_{s}}{\left(a_{0}+a_{1}\right) D} z=\frac{a_{1}}{a_{0}+a_{1}} \ddot{y}+\frac{a_{2} U}{\left(a_{0}+a_{1}\right) D} \dot{y} .
$$

We may recall the experimental observation (which was already used earlier) that the natural frequency of the fluid oscillations is equal to $\omega_{s}$; thus the coefficient of $z$ in (2.14) can be replaced by $\omega_{s}^{2}$.

The motion of the cylinder is modeled by a damped oscillator forced by the fluid, namely

$$
m \ddot{y}+r \dot{y}+k y=F_{y}
$$

with $m>0$ the mass per unit length of the cylinder, $r \geq 0$ the structural damping, and $k \geq 0$ the support stiffness. Inserting the expression for the force in (2.11) into (2.15) yields the following second-order equation for the cylinder motion:

$$
\ddot{y}+\frac{r+a_{2} \rho D U}{m+a_{1} \rho D^{2}} \dot{y}+\frac{k}{m+a_{1} \rho D^{2}} y=\frac{a_{1} \rho D^{2}}{m+a_{1} \rho D^{2}} \ddot{z}+\frac{a_{2} \rho D U}{m+a_{1} \rho D^{2}} \dot{z} .
$$

The pair (2.14) and (2.16) are the equations of motion for the coupled fluid-structure oscillations.

3. Rescaling to the Singular Perturbation. The goal of this paper is to examine the limit behavior of solutions of the coupled equations (2.14) and (2.16), for high velocity $U$ and small viscosity, namely small $a_{2}$ (see (2.11)). As noted earlier, the frequency of the vortex shedding $\omega_{s}$ and the velocity of the vortex street $u_{T}$ are of the same order of magnitude as $U$. As a preparation for the rescaling, we copy equations (2.14) and (2.16), maintaining the dependence on the variables $a_{2}$ and $U$ while replacing the other coefficients with constants of a simpler form:

$$
\begin{gathered}
\ddot{z}+\gamma_{1} a_{2} U \dot{z}-\gamma_{2} U^{2} F\left(\frac{\dot{z}}{U}\right)+\gamma_{3} u_{T} \omega_{s} z=\gamma_{4} \ddot{y}+\gamma_{5} a_{2} U \dot{y} \\
\ddot{y}+\left(\delta_{1}+\delta_{2} a_{2} U\right) \dot{y}+\delta_{3} y=\delta_{4} \ddot{z}+\delta_{5} a_{2} U \dot{z}
\end{gathered}
$$


where the coefficients can be recovered by a comparison to (2.14) and (2.16) and are as follows. We set

$$
\gamma_{0}=\left(a_{0}+a_{1}\right)^{-1}, \quad \delta_{0}=\left(m+a_{1} \rho D^{2}\right)^{-1} .
$$

Then

$$
\begin{gathered}
\gamma_{1}=\frac{1}{D} \gamma_{0}, \quad \gamma_{2}=\frac{K_{1}}{\rho D^{2}} \gamma_{0}, \quad \gamma_{3}=\frac{K}{D} \gamma_{0}, \quad \gamma_{4}=a_{1} \gamma_{0}, \quad \gamma_{5}=\frac{1}{D} \gamma_{0}, \\
\delta_{1}=r \delta_{0}, \quad \delta_{2}=\rho D \delta_{0}, \quad \delta_{3}=k \delta_{0}, \quad \delta_{4}=a_{1} \rho D^{2} \delta_{0}, \quad \delta_{5}=\rho D \delta_{0} .
\end{gathered}
$$

Note that all the coefficients $\gamma_{i}$ and $\delta_{i}$ are nonnegative.

Since $\gamma_{4}<1$ and $\delta_{4}<1$, it follows that $1-\gamma_{4} \delta_{4}$ is positive. This helps us in the next manipulation as follows. We multiply Eq. (3.1) by $\delta_{4}$ and add to Eq. (3.2), and multiply Eq. (3.2) by $\gamma_{4}$ and add to Eq. (3.1). After rearranging terms and dividing through by $\left(1-\gamma_{4} \delta_{4}\right)$ we get the following:

$$
\begin{gathered}
\ddot{z}+c\left(\gamma_{1}-\gamma_{4} \delta_{5}\right) a_{2} U \dot{z}-c \gamma_{2} U^{2} F\left(\frac{\dot{z}}{U}\right)+c \gamma_{3} u_{T} \omega_{s} z \\
=c\left(-\delta_{1} \gamma_{4}+\left(\gamma_{5}-\gamma_{4} \delta_{3}\right) a_{2} U\right) \dot{y}-c \gamma_{4} \delta_{3} y \\
\ddot{y}+c\left(\delta_{1}+\left(\delta_{2}-\gamma_{5} \delta_{4}\right) a_{2} U\right) \dot{y}+c \delta_{3} y \\
=c\left(\delta_{5}-\gamma_{1} \delta_{4}\right) a_{3} U \dot{z}+\gamma_{2} \delta_{4} U^{2} F\left(\frac{\dot{z}}{U}\right)-\gamma_{3} \delta_{4} u_{T} \omega_{s} z
\end{gathered}
$$

with $c=\left(1-\gamma_{4} \delta_{4}\right)^{-1}$.

At this point we carry out the rescaling to the singular perturbation form.

We choose a typical velocity $\bar{U}$ and set $U=\varepsilon^{-1} \bar{U}$. In accordance with the previous observations, we also have then $u_{T}=\varepsilon^{-1} \bar{u}_{T}$ and $\omega_{s}=\varepsilon^{-1} \bar{\omega}_{s}$ for the induced typical translation velocity and frequency of the vortex street and vortex shedding.

Concerning the singular variable $a_{2}$ we follow two options:

Case I: $a_{2}$ is inversely proportional to the velocity of the fluid. We then write $a_{2}=\varepsilon \bar{a}_{2}$.

Case II: $a_{2}$ is of order smaller than the inverse of the velocity. We model this case by setting, say, $a_{2}=\varepsilon^{2} \bar{a}_{2}$.

Next we make the following change of variables:

$$
\theta_{1}=z, \quad \theta_{2}=\varepsilon \dot{z}, \quad x_{1}=\varepsilon^{2} y, \quad x_{2}=\varepsilon^{2} \dot{y} .
$$

Inserting the variables defined in (3.7) into (3.5) and (3.6), we get the following equations: Case I: Viscosity term inversely proportional to the fluid velocity.

$$
\begin{aligned}
\dot{x}_{1} & =x_{2}, \\
\dot{x}_{2} & =-\alpha_{1} x_{1}-\alpha_{2} x_{2}-\beta_{3} \theta_{1}+\beta_{4} F\left(\gamma \theta_{2}\right)+\varepsilon \beta_{5} \theta_{2}, \\
\varepsilon \dot{\theta}_{1} & =\theta_{2}, \\
\varepsilon \dot{\theta}_{2} & =-\beta_{1} \theta_{1}+\beta_{2} F\left(\gamma \theta_{2}\right)-\varepsilon \beta_{6} \theta_{2}-\alpha_{3} x_{1}-\alpha_{4} x_{2}
\end{aligned}
$$


where

$$
\begin{aligned}
& \alpha_{1}=c \delta_{3}, \quad \alpha_{2}=c\left(\delta_{1}+\left(\delta_{2}-\gamma_{5} \delta_{4}\right) \bar{a}_{2} \bar{U}\right), \quad \alpha_{3}=c \gamma_{4} \delta_{3}, \\
& \alpha_{4}=c\left(\gamma_{4} \delta_{1}-\left(\gamma_{5}-\gamma_{4} \delta_{2}\right) \bar{a}_{2} \bar{U}\right), \quad \beta_{1}=c \gamma_{3} \bar{u}_{T} \bar{\omega}_{s}, \\
& \beta_{2}=c \gamma_{2} \bar{U}^{2}, \quad \beta_{3}=c \gamma_{3} \delta_{4} \bar{u}_{T} \bar{\omega}_{s} . \quad \beta_{4}=c \gamma_{2} \delta_{4} \bar{U}^{2}, \\
& \beta_{2}=c\left(\delta_{5}-\gamma_{1} \delta_{4}\right) \bar{a}_{2} \bar{U}, \quad \beta_{6}=c\left(\gamma_{1} \gamma_{4} \delta_{5}\right) \bar{a}_{2} \bar{U}, \quad \gamma=\frac{1}{\bar{U}}, \quad c=\left(1-\gamma_{4} \delta_{4}\right)^{-1}
\end{aligned}
$$

and where the values of $\gamma_{i}, \delta_{i}$ are given in (3.3) and (3.4).

Case II: Viscosity term smaller than inversely proportional to the fluid velocity.

$$
\begin{aligned}
\dot{x}_{1} & =x_{2}, \\
\dot{x}_{2} & =-\alpha_{1} x_{1}-\left(\alpha_{2}^{\prime}+\varepsilon \alpha_{5}\right) x_{2}-\beta_{3} \theta_{1}+\beta_{4} F\left(\gamma \theta_{2}\right)+\varepsilon \beta_{5} \theta_{2}, \\
\varepsilon \dot{\theta}_{1} & =\theta_{2}, \\
\varepsilon \dot{\theta}_{2} & =-\beta_{1} \theta_{1}+\beta_{2} F\left(\gamma \theta_{2}\right)-\varepsilon^{2} \beta_{6} \theta_{2}-\alpha_{3} x_{1}-\left(\alpha_{4}^{\prime}+\varepsilon \alpha_{6}\right) x_{2},
\end{aligned}
$$

where the coefficients are as in $(3.10)$ with the addition of

$$
\alpha_{2}^{\prime}=c \delta_{1}, \quad \alpha_{4}^{\prime}=c \gamma_{4} \delta_{1}, \quad \alpha_{5}=c\left(\delta_{2}-\gamma_{5} \delta_{4}\right) \bar{a}_{2} \bar{U}, \quad a_{6}=c\left(\gamma_{5}-\gamma_{4} \delta_{2}\right) \bar{a}_{2} \bar{U}
$$

4. Solving the Singularly Perturbed System. For the analysis of the solutions of the system (3.8)-(3.9), note that the terms incorporating a regular perturbation will have as $\varepsilon \rightarrow 0$ a negligible effect on a finite time interval. We therefore copy here the equations, suppressing however these terms:

$$
\begin{aligned}
\dot{x}_{1} & =x_{2}, \\
\dot{x}_{2} & =-\alpha_{1} x_{1}-\alpha_{2} x_{2}-\beta_{3} \theta_{1}+\beta_{4} F\left(\gamma \theta_{2}\right), \\
\varepsilon \dot{\theta}_{1} & =\theta_{2}, \\
\varepsilon \dot{\theta}_{2} & =-\beta_{1} \theta_{1}+\beta_{2} F\left(\gamma \theta_{2}\right)-\alpha_{3} x_{1}-\alpha_{4} x_{2} .
\end{aligned}
$$

Notice that the analogous limit equations for Case II, namely the modification of (3.11)(3.12), are almost the same as the pair (4.1)-(4.2) with the difference being replacing $\alpha_{2}$ by $\alpha_{2}^{\prime}$ and $\alpha_{4}$ by $\alpha_{4}^{\prime}$. The analysis in this section does not use the specific values of the coefficients, except that $\beta_{1}, \beta_{2}$, and $\gamma$ are positive and $F$ satisfies Assumption 2.1. In the next section we comment on the consequences of the theory for the specific values of the parameters in the two cases.

The system (4.1)-(4.2) represents coupled slow and fast oscillators and has the standard form commonly encountered in the literature (see O'Malley [10, Chapter 2(D)]). However, the standard method employed in the literature, namely the Levinson-Tichonov approach, does not apply to (4.1)-(4.2). Indeed, the method suggests that the limit as $\varepsilon \rightarrow 0$ of solutions of (4.1)-(4.2) is captured by solutions of the differential-algebraic system obtained when $\varepsilon=0$ is set in (4.2). For the method to work, the solutions of the algebraic equation should be asymptotically stable equilibria for the fast dynamics on the fast time scale. This may not be the case for the system (4.1)--(4.2), and in fact it is definitely not the case when $F(\eta)$ is taken to be either of the correction terms (2.6) 
or (2.7) suggested by Iwan and Belvins [5] or Dowell and Ilgamov [4] (see Remark 2.2). Indeed $F^{\prime}(0)>0$ makes an equilibrium of the fast dynamics unstable.

To cope with the dynamics of (4.1)-(4.2) we employ the method introduced in [1], which enables the analysis of fast dynamics that may not converge to a stationary point.

The first step is to examine the fast dynamics on the fast time scale, namely the system

$$
\begin{aligned}
& \dot{\theta}_{1}=\theta_{2}, \\
& \dot{\theta}_{2}=-\beta_{1} \theta_{1}+\beta_{2} F\left(\gamma \theta_{2}\right)+c\left(x_{1}, x_{2}\right)
\end{aligned}
$$

where $c\left(x_{1}, x_{2}\right)=-\alpha_{3} x_{1}-\alpha_{4} x_{2}$ is regarded as a constant. Recall that $\beta_{1}, \beta_{2}$, and $\gamma$ are positive (see (3.10) and (3.3)) and that $F$ satisfies Assumption 2.1.

Lemma 4.1. Solutions of (4.3) are attracted, as $t \rightarrow \infty$, to a bounded set in the $\left(\theta_{1}, \theta_{2}\right)$ plane; the set is uniformly bounded for a bounded set of pairs $\left(x_{1}, x_{2}\right)$.

Proof. We reduce the problem to the classical Cartwright-Littlewood equation of the form

$$
\ddot{\zeta}-\gamma \beta_{2} f(\gamma \zeta) \dot{\zeta}+\beta_{1} \zeta=0
$$

where $f(\zeta)=F^{\prime}(\zeta)$. The conditions in Assumption 2.1 imply that the conditions of Theorem 8.1 in Lefschetz [8, XI.4] are satisfied, hence by that theorem, solutions of (4.4) converge to a bounded set in the $(\zeta, \dot{\zeta})$-plane. Consider the variable $\theta$ given by

$$
\beta_{1} \theta=\dot{\zeta}-\beta_{2} F(\gamma \zeta)+c\left(x_{1}, x_{2}\right)
$$

The variable $\theta$ satisfies $\beta_{1} \dot{\theta}=\ddot{\zeta}-\gamma \beta_{2} f(\gamma \zeta) \dot{\zeta}$, and hence by (4.4)

$$
\dot{\theta}=-\zeta \text {. }
$$

Inserting $\zeta=-\dot{\theta}, \dot{\zeta}=-\ddot{\theta}$ into (4.5) yields

$$
\beta_{1} \theta=-\ddot{\theta}-\beta_{2} F(-\gamma \dot{\theta})+c\left(x_{1}, x_{2}\right)
$$

and since $F$ is an odd function equation, it follows that (4.7) is identical to the system (4.3) with $\theta=\theta_{1}$ and $\dot{\theta}=\theta_{2}$. Since solutions of (4.4) are attracted to a bounded set in the $(\zeta, \dot{\zeta})$-plane, so are (by $(4.5))$ solutions of $(4.3)$. This completes the proof.

LEMMA 4.2. Each solution of (4.3) converges to a periodic orbit, and each periodic orbit is symmetric around the equilibrium $\left(\theta_{1}, \theta_{2}\right)=\left(\beta_{1}^{-1} c\left(x_{1}, x_{2}\right), 0\right)$; namely, the mapping $\left(\theta_{1}, \theta_{2}\right) \rightarrow\left(\beta_{1}^{-1} c\left(x_{1}, x_{2}\right)-\left(\theta_{1}-\beta_{1}^{-1} c\left(x_{1}, x_{2}\right)\right),-\theta_{2}\right)$ maps the periodic orbit into itself.

Proof. Once the boundedness is established in the previous lemma, the convergence follows from the Poincaré-Bendixson Theorem. For the second statement, notice that since $F$ is an odd function, then if $\left(\beta_{1}^{-1} c\left(x_{1}, x_{2}\right)+\theta_{1}(t), \theta_{2}(t)\right)$ is a solution, so is $\left(\beta_{1}^{-1} c\left(x_{1}, x_{2}\right)-\theta_{1}(t),-\theta_{2}(t)\right)$. This symmetry applies also to the periodic orbits. Since a periodic orbit around a point must intersect its reflection with respect to the point, uniqueness of solutions implies that the periodic orbit of (4.3) coincides with its reflection with respect to $\left(\beta_{1}^{-1} c\left(x_{1}, x_{2}\right), 0\right)$. This completes the proof. 
For the next observation, recall the notion of an invariant measure of a differential equation; see Nemytskii and Stepanov [9], or see [1]. All invariant measures considered in this paper are invariant probability measures, namely, they are nonnegative and the measure of the whole space $\mathbb{R}^{2}$ is 1 . Uniqueness of solutions implies that any periodic orbit supports a unique invariant probability measure.

LEMma 4.3. Each invariant probability measure of (4.3) is supported on the collection of periodic orbits (including the equilibrium) of (4.3). Furthermore, any invariant measure $\mu$ of $(4.3)$ is the direct integral

$$
\int \mu_{q} \nu(d q)
$$

where $q$ is a parameterization of the periodic orbits, $\nu(d q)$ is a probability measure on the variable $q$, and $\mu_{q}$ is the unique invariant measure supported on $q$. (Here, naturally, $\left(\int \mu_{q} \nu(d q)\right)(B)=\int \mu_{q}(B) \nu(d q)$.)

Proof. The convergence established in Lemma 4.1 implies that the invariant measures must be supported on the periodic orbits. The direct integral decomposition follows from the general decomposition result for invariant measures of differential equations due to Kryloff and Bogoliouboff; see Nemytskii and Stepanov [9, p. 497]; it follows since the measures supported on a periodic orbit form the extreme points of all invariant probability measures.

Corollary 4.4. Let $\mu=\mu\left(d \theta_{1} \times d \theta_{2}\right)$ be an invariant probability measure of (4.3). Then

$$
\int \theta_{1} d \mu=\beta_{1}^{-1} c\left(x_{1}, x_{2}\right), \quad \int F\left(\gamma \theta_{2}\right) d \mu=0 .
$$

Proof. The result follows from Lemma 4.3, Lemma 4.2, and the oddness of $F$ required in Assumption 2.1.

With the preparations made in the preceding results, the technique in [1] can be applied. To characterize the limit behavior of solutions of (4.1)-(4.2) as $\varepsilon \rightarrow 0$ we consider the fast solution $\theta_{\varepsilon}(t)=\left(\theta_{1}(t), \theta_{2}(t)\right)_{\varepsilon}$ as a Dirac measure-valued function, namely, mapping $t$ into the probability measure supported on $\left\{\theta_{\varepsilon}(t)\right\}$. We consider Young measures as possible limits as $\varepsilon \rightarrow 0$ of the function $\theta_{\varepsilon}$, namely, functions $\mu_{t}$ that map $t$ into the family of probability measures on $\mathbb{R}^{2}$. The Young-measures convergence (called statistical convergence in [1]) of $\theta_{i}(t)$ to $\mu_{t}$ on $[a, b]$ is characterized by the convergence of

$$
\int_{a}^{b} h\left(t, \theta_{i}(t)\right) d t \rightarrow \int_{a}^{b} \int_{\mathbb{R}^{2}} h(t, \theta) \mu_{t}(d \theta) d t
$$

for every $h:[a, b] \times \mathbb{R}^{2} \rightarrow \mathbb{R}$ that is continuous and bounded; see, e.g., [1], or Ball [2]. Alternatively, statistical convergence reflects that the distributions of the mappings $t \rightarrow\left(t, \theta_{i}(t)\right)$ converge to the distribution on $\mathbb{R}^{2}$ of the mapping $t \rightarrow\left(t, \mu_{t}\right)$. It is clear that in our case, if $\theta_{i}(t)$ converge as Young measures to $\mu_{t}$, then for a point $t_{0}$ at which the map $t \rightarrow \mu_{t}$ is continuous, the behavior of a trajectory $\theta_{i}(\cdot)$ in a small interval $\left(t_{0}-\delta, t_{0}+\delta\right)$ is depicted approximately by the invariant measure $\mu_{t_{0}}$. 
In what follows, $x=\left(x_{1}, x_{2}\right)$ and $\theta=\left(\theta_{1}, \theta_{2}\right)$.

ThEOREM 4.5. Let $\left(x_{\varepsilon}(t), \theta_{\varepsilon}(t)\right)$ solve (4.1)-(4.2) with a fixed initial condition $x_{\varepsilon}(0)=\bar{x}$ and initial conditions $\theta_{\varepsilon}(0)$ in a bounded domain of $\mathbb{R}^{2}$. On any finite time interval $\left[0, T_{0}\right]$, the trajectories $x_{\varepsilon}(t)$ converge uniformly as $\varepsilon \rightarrow 0$ to the solution of

$$
\begin{aligned}
& \dot{x}_{1}=x_{2}, \\
& \dot{x}_{2}=-\alpha_{1} x_{1}-\alpha_{2} x_{2}-\beta_{3} \beta_{1}^{-1} c\left(x_{1}, x_{2}\right)
\end{aligned}
$$

with initial condition $x(0)=\bar{x}$. The solutions $\theta_{\varepsilon}(t)$ converge as Young measures to the family of maps $t \rightarrow \mu_{t}$ where for each $t$ the value $\mu_{t}$ is an invariant measure of (4.3) with $c\left(x_{1}, x_{2}\right)=c\left(x_{1}(t), x_{2}(t)\right)$, when $\left(x_{1}(t), x_{2}(t)\right)$ is the solution of (4.11).

Proof. Given Corollary 4.4 and Lemmas 4.1, 4.2, 4.3, the result is an application of Theorem 2.2 in [1]. For completeness we outline the procedure that leads to the result. First, invariant measures of the fast flow on the fast time scale should be computed when the slow variable is considered as constant. This is the outcome of Lemma 4.3. The fast flow must also be bounded, as Lemma 4.1 guarantees. Then the terms containing the fast variable in the slow equation must be averaged according to each invariant measure. In our case the result is independent of the invariant measure as given by Corollary 4.4 , and the average of $F\left(\gamma \theta_{2}\right)$ is 0 while the average of $-\beta_{3} \theta_{1}$ is $-\beta_{3} \beta_{1}^{-1} c\left(x_{1}, x_{2}\right)$. The latter term determines the limit differential equation for the slow variable as given in (4.11). Then the Young measure convergence follows as verified in [1].

5. Conclusions. In this section we draw the consequences of the limit analysis performed in the previous section. We examine several possibilities of the coefficients, especially the correction term $F(\eta)$. We start with the effective motion of the cylinder, continue with an analysis of a control applied to the cylinder, and conclude with the analysis of the fluid oscillations.

5.1. Effective dynamics of the cylinder. Representing Eq. (4.11), which captures the limit behavior of the cylinder's oscillations, as a second-order equation for $x=x_{1}$, we get

$$
\ddot{x}+\left(\alpha_{1}-\beta_{3} \beta_{1}^{-1} \alpha_{3}\right) \dot{x}+\left(\alpha_{2}-\beta_{3} \beta_{1}^{-1} \alpha_{4}\right) x=0
$$

for Case I, where viscosity is inversely proportional to the fluid velocity. In Case II, where viscosity is of small order, the equations are similar, with the difference being that $\alpha_{2}^{\prime}$ and $\alpha_{4}^{\prime}$ replace $\alpha_{2}$ and $\alpha_{4}$.

For general parameters $\alpha_{i}$ and $\beta_{i}$, Eq. (5.1) may be unstable, even if the original uncoupled oscillators are both stable. This is not the case in the fluid-structure coupling. Indeed, for Case I represented in (5.1), tracking back the values of the coefficients through (3.10), (3.4), and (3.3) yields

$$
\left(m+a_{1} \rho D^{2}\right) \ddot{x}+\left(r+\rho D \bar{a}_{2} \bar{U}\right) \dot{x}+k x=0 .
$$

For Case II, where viscosity is of lower order, the limit equation is

$$
\left(m+a_{1} \rho D^{2}\right) \ddot{x}+r \dot{x}+k x=0 .
$$


Comparison with the original cylinder equation of motion $(2.15)$ (recall that $x=\varepsilon^{2} y$ ) reveals that the coupling with the fluid induces an effective new mass

$$
m+a_{1} \rho D^{2}
$$

while the other coefficients stay put except for an addition of $\rho D \bar{a}_{2} \bar{U}$ to the damping coefficient when viscosity is inversely proportional to the fluid velocity. We therefore conclude that in the case (5.3), where viscosity is of order smaller than inversely proportional to the fluid velocity, the effect of the fluid on the cylinder in the singular perturbation limit is the reduction of the stabilizing attraction forces. Indeed, the heavier mass shifts the eigenvalues toward the unstable domain. In the case (5.2), where viscosity is inversely proportional to the fluid velocity, the reduction in the stability forces due to a heavier mass may be compensated by the increase of the effective damping of the structure.

5.2. Effective control force. Consider a control force applied to the structure, say in an attempt to excite or to suppress oscillations. Suppose it takes the form of a forcing term in Eq. (2.15), namely,

$$
m \ddot{y}+r \dot{y}+k y=F_{y}+W(t) .
$$

We examine what the effective control force is in the singular limit as $U \rightarrow \infty$ and $a_{2} \rightarrow 0$ as in the previous analysis. Following carefully the previous analysis and using the rescaling

$$
w(t)=\varepsilon^{2} W(t)
$$

and employing the assumption that $W(t)$ is defined on the slow time scale, one gets as the effective equations in Cases I and II, respectively, the equations

$$
\left(m_{1}+a_{1} \rho D^{2}\right) \ddot{x}+\left(r+\rho D \bar{a}_{2} \bar{U}\right) \dot{x}+k x=w(t)
$$

and, respectively,

$$
\left(m_{1}+a_{1} \rho D^{2}\right) \ddot{x}+r \dot{x}+k x=w(t) .
$$

We leave out the detailed derivations. We conclude that the control action is modulated according to the effective mass determined in (5.4), and possibly according to the increase of damping in the case of (5.7).

5.3. Effective fluid oscillations. Here the conclusions depend on the form of the correction term $F(\eta)$ in Assumption 2.1. We focus on two possibilities as follows.

5.3.1. $\quad F^{\prime}(0)<0$ and $F(\eta)<0$ for $\eta>0$.

In this case the fast motion (4.3) is actually asymptotically stable and all its solutions converge to the equilibrium $\left(\beta_{3} \beta_{1}^{-1}\left(-\alpha_{3} x_{1}-\alpha_{4} x_{2}\right), 0\right)$. The equation of the cylinder is the same as before; hence we conclude that the fast dynamics in the singular limit oscillates at the same pace as the cylinder, namely (recall that $\theta(t)=z(t)$, see (3.7))

$$
\theta(t)=-\delta_{4} \alpha_{3} x(t)-\delta_{4} \alpha_{4} \dot{x}(t)
$$

and

$$
\theta(t)=-\delta_{4} \alpha_{3} x(t)-\delta_{4} \alpha_{4}^{\prime} \dot{x}(t)
$$


in Case I and Case II, respectively, when $x(t)$ is the solution of (5.2) and (5.3), respectively. The exact form of the coefficients can be traced back through (3.10), (3.4), and (3.3). We note that in this case one does not need the invariant measures analysis performed in Sec. 4, since the Levinson-Tichonov method can be applied. Indeed, the only invariant measure of (4.3) is at the equilibrium, and $\theta_{\varepsilon}(t)$ converges as $\varepsilon \rightarrow 0$ to the limit function in (5.9) or, respectively, (5.10), pointwise except possibly on a boundary layer near $t=0$.

\subsection{2. $\quad F^{\prime}(\eta)>0$.}

This is the case suggested by Iwan and Belvins [5] and Dowell and Ilgamov [4]; see Remark 2.2. In this case the equilibrium of (4.3) is unstable (and, in particular, the Levinson-Tichonov approach is not applicable). An invariant measure may still be supported on the equilibrium, but the instability of the origin implies that the actual physical dynamics will tend to periodic orbits. These periodic orbits are bounded away from the equilibrium. There may be a unique periodic orbit as in the case of (2.6) that is equivalent to the van der Pol oscillator as Lemma 4.1 shows. Then at any time $t$ the fast variable will exhibit nontrivial fast oscillations around the periodic orbit (or around some of them in the case of multiple periodic orbits). The center of the oscillations in each case is, however, given by the right-hand side of (5.9) and, respectively, (5.10); namely, the fluid will exhibit fast oscillations centered around a point that oscillates at the same pace as the cylinder.

\section{REFERENCES}

[1] Z. Artstein and A. Vigodner, Singularly perturbed ordinary differential equations with dynamic limits, Proc. Royal Society Edinburgh 126A, 541-569 (1996)

[2] J. M. Ball, A version of the fundamental theorem for Young measures, in PDEs and Continuum Models of Phase Transitions, M. Rascle, D. Serre, and M. Slemrod, eds., Lecture Notes in Physics, 344, Springer-Verlag, Berlin-New York, 1988, pp. 207-215

[3] Y. N. Chen, Fluctuating lift forces of the Kármán vortex streets on single circular cylinders and in tube bundles, Part 1, J. of Engineering for Industry, Trans. ASME 96, Ser. B, 603-612 (1972)

[4] E. H. Dowell and M. Ilgamov, Studies in Nonlinear Aeroelasticity, Springer-Verlag, New York, 1988

[5] W. D. Iwan and R. D. Belvins, A model for vortex induced oscillation of structure, J. Applied Mechanics 41, 581-586 (1974)

[6] Th. von Kármán, Über den Mechanismus des Flüssigkeitswiderstandes den ein bewegter Körper in einer Flüssigkeit erfährt, Nachrichten Königlichen Gesellschaft der Wissenschaften zu Göttingen, 1912, pp. 508-517

[7] N. E. Kochin, I. A. Kibel', and N. V. Roze, Theoretical Hydromechanics, John Wiley and Sons, New York, 1964

[8] S. Lefschetz, Differential Equations: Geometric Theory, Dover, New York, 1977

[9] V. V. Nemytskii and V. V. Stepanov, Qualitative Theory of Differential Equations, Princeton University Press, Princeton, 1960

[10] R. E. O'Malley, Jr., Singular Perturbation Methods of Ordinary Differential Equations, SpringerVerlag, New York, 1991 